\title{
A study on the relationship between internal nozzle geometry and injected mass distribution of eight ECN Spray G nozzles
}

\author{
K.E. Matusik ${ }^{1, *}$, D.J. Duke ${ }^{1,2}$, N. Sovis ${ }^{1}$, A.B. Swantek ${ }^{1}$, C.F. Powell ${ }^{1}$, R. Payri ${ }^{3}$, D. Vaquerizo ${ }^{3}$, \\ S. Giraldo-Valderrama ${ }^{3}$, A.L. Kastengren ${ }^{4}$ \\ ${ }^{1}$ Energy Systems Division, Argonne National Laboratory, Lemont, IL, USA \\ ${ }^{2}$ Laboratory for Turbulence Research in Aerospace \& Combustion, Department of Mechanical \\ \& Aerospace Engineering, Monash University, Australia \\ ${ }^{3} \mathrm{CMT}$ Motores Termicos, Universitat Politècnica de València, València, Spain \\ ${ }^{4}$ X-Ray Science Division, Argonne National Laboratory, Lemont, IL, USA \\ *Corresponding author: kmatusik@anl.gov
}

\begin{abstract}
Gasoline direct injection (GDI) nozzles are manufactured to meet geometric specifications with length scales on the order of a few hundred microns. The machining tolerances of these nominal dimensions are not always known due to the difficulty in accurately measuring such small length scales in a nonintrusive fashion. To gain insight into the variability of the machined dimensions as well as any effects that this variability may have on the fuel spray behavior, a series of measurements of the internal geometry and fuel mass distribution were performed on a set of eight nominally duplicate GDI "Spray G" nozzles provided by the Engine Combustion Network. The key dimensions of each of the eight nozzle holes were measured with micron resolution using full spectrum $x$-ray tomographic imaging at the 7-BM beamline of the Advanced Photon Source at Argonne National Laboratory. Fuel density distributions at $2 \mathrm{~mm}$ downstream of the nozzle tips were obtained by performing $x$-ray radiography measurements for many lines of sight. The density measurements reveal nozzle-to-nozzle as well as hole-to-hole density variations. The combination of high-resolution geometry and fuel distribution datasets allows spray phenomena to be linked to specific geometric characteristics of the nozzle, such as variability in the hole lengths and counterbore diameters, and the hole inlet corner radii. This analysis provides important insight into which geometrical characteristics of the nozzles may have the greatest importance in the development of the injected sprays, and to what degree these geometric variations might account for the total spray variability. The goal of this work is then to further the understanding of the relationship between internal nozzle geometry and fuel injection, provide input to improve computational models, and ultimately aid in optimizing injector design for higher fuel efficiency and lower emissions engines.
\end{abstract}

\section{Keywords}

GDI, nozzle geometry, fuel spray density, fuel injector, DISI, Spray G, ECN, gasoline

\section{Introduction}

To fully realize the benefits of a gasoline direct injection (GDI) engine, precise control over the amount of injected fuel and the fuel-to-air mixing ratio is necessary. Any variations to the fuel spray distribution can affect the combustion process, and by extension the fuel efficiency and emissions levels. Modern multi-hole GDI nozzles generally feature a relatively complex step-hole geometry in which each hole might require knowledge of at least nine dimensions to accurately model. Due to the importance of the injection process on the output metrics of a GDI engine, a subset of internal combustion engine research has been dedicated to investigating the effects of nozzle geometry on the corresponding fuel spray distribution. X-ray spray tomography measurements of three six-hole GDI nozzles with varying hole patterns explored the effects of geometric asymmetries on the spray structure [1]. The authors found that the emitted fuel spray distribution varied between jet-like, hollow-coned, or crescent-shaped, depending on the nozzle hole pattern. A study using a single-cylinder optical engine reported higher soot emissions for a laser-drilled GDI injector in comparison to one manufactured using electrical discharge machining [2]. Research has also focused on elucidating the relationship between nozzle shape and cavitation, whose presence can significantly perturb fuel spray characteristics such as the discharge coefficient, outlet velocity, spray angle, and atomization behavior [3]. A converging diameter profile and a sufficiently rounded hole inlet corner were found to decrease or eliminate geometric cavitation in comparison to a sharp-cornered, cylindrical geometry $[4,5]$. In addition, local asymmetries at the hole inlet have been shown to generate correspondingly asymmetric cavitation, forming at the location where the fuel flow experiences the largest change in direction [5].

Research in the same vein has been carried out in collaboration with the Engine Combustion Network (ECN). The ECN coordinates the sharing of well-characterized gasoline and diesel injection nozzles and standardized hardware with experimental facilities worldwide. These facilities perform various diagnostics at established conditions and share their findings publicly, with the intent of building a comprehensive database of experimental and computational measurements of the fuel injection and combustion processes. The ECN community's target injector for GDI studies is "Spray G", an eight-hole solenoid-activated valve-covered orifice injector with a step-hole geometry [6]. A number of nominally duplicate Spray G injectors, as identified by the last two digits of their respective serial numbers, 
have been in circulation at multiple facilities for experimental diagnostics. Nozzle geometry measurements of Spray G \#28 exposed 7\% variability in hole area and a factor of two variability in the inlet corner radius. Complementary spray tomography measurements showed significant plume-to-plume mass flux variations [7]. Simulation results for Spray G using nominal geometry have revealed strong asymmetric transients in the internal flow structure [8], which are expected to respond to geometric asymmetries such as an azimuthally-varying inlet hole corner radius. These findings support an inextricable link between the internal nozzle geometry and the fuel spray distribution, and as such, the need to expose which geometric features are responsible for spray variability. In addition, an understanding of spray sensitivity to geometric variations can provide more educated tolerances to manufacturing designers.

A number of experimental measurements have been executed using Spray G hardware. Optical techniques were implemented to investigate fuel spray characteristics [9]. Schlieren and diffused back illumination were used to characterize the vapor and liquid penetration [10], shadowgraphy and Mie scattering have provided spray visualization [11], and phase Doppler anemometry has been used to measure fuel droplet sizes and velocities [11, 12], to name a few. Intrusive techniques, such as patternation, can provide a footprint of the spray mass distribution [13]. Rate of injection and rate of momentum measurements have also been successfully demonstrated on Spray G \#26 [14].

To isolate hole-to-hole spray variations, measurements must be made in a noninvasive manner and near to the nozzle exit before plume interactions significantly influence the density field. These dense regions of the spray do not lend themselves well to optical techniques on account of multiple scattering which causes short optical depths. At low energies, $x$-rays are only weakly scattered by the spray, with photoelectric absorption instead being their main mode of interaction with the fuel [15]. As such, x-rays generated by a benchtop or synchrotron source are useful in obtaining quantitative density measurements in multiphase flows [16, 17]. A benchtop x-ray system coupled with polycapillary optics has been previously used to obtain density measurements of the fuel spray emitted by a six-hole GDI injector [18]. In general, the inherent divergence of the x-ray source limits the spatial resolution, and the lower flux limits the temporal resolution and signal-to-noise ratio. The high photon flux of a third-generation synchrotron source, such as the Advanced Photon Source (APS) at Argonne National Laboratory, provides the sub-microsecond temporal and micron spatial resolution that is desired for investigating fuel sprays. The 7-BM beamline at the APS is tailored for such experiments, including time-resolved measurements of the ensemble-averaged projected fuel density with micron spatial resolution [19], as well as shot-to-shot statistics [20]. Measuring the projected density at a few lines of sight is useful for modeling the density field of simple sprays such as those from a single-hole nozzle [21]. However, for a more detailed quantification of the density distribution of a more complicated spray structure such as one from a multi-hole injector, a full tomographic reconstruction can be implemented [1, 7, 22, 23].

There exist various methods for measuring the internal nozzle geometry. Depending on the shape, the dimensions of a silicone mold of the hole can measured with a scanning electron microscope [24]. Single-micron resolution of geometric features is possible using optical microscopy, but the technique is limited by the depth of focus and location of the minimum hole diameter, past which measurements cannot be made [25]. Computed tomography (CT) can be implemented to obtain the nozzle geometry by making use of x-rays and scanning the object for a wide range of angles. Tomographically reconstructing the projection images provides a full three-dimensional representation of the scanned section of the nozzle. A benchtop x-ray source may be used to generate the requisite $x$-rays, but, once again, the angular divergence of the beam limits the spatial resolution. Sub-micron resolution with a benchtop source is possible, but is generally reserved for low-density materials [26]. Synchrotron sources, such as the APS and the European Synchrotron Radiation Facility, for example, are particularly well suited for CT measurements due to their highly brilliant $\mathrm{x}$-ray sources, and each maintains a facility that specializes in x-ray microtomography measurements of low-density samples $[27,28]$. Within the last few years, a new instrument has been added to the 7-BM beamline at the APS that allows for high-resolution CT imaging of injection nozzles. With this complementary capability, the 7-BM facility now supports measurements of the nozzle geometry as well as the density field with minor modifications to the experimental setup.

The following sections outline the experimental setup for performing nozzle tomography and spray tomography experiments at the 7-BM beamline. We summarize the methodology followed to obtain the dimensions of eight Spray G nozzles, as well as the time-resolved density field at $2 \mathrm{~mm}$ downstream of the nozzle tip. We then provide results from multiple linear regression analysis that couples the effects of geometric features on the steady-state density field. These findings highlight the key players that may be responsible for spray variability, and provide a prioritized list of geometric features that may be critical for accurate representation in future computational efforts, as well as machining with high precision during the injector manufacturing process.

\section{Material and methods}

All experiments were performed at the 7-BM beamline of the APS [29]. The eight Spray G injectors that contributed to this study are numbers $12,17,18,21,22,25,26$, and 29 . The coordinate system that is used to describe the nozzle geometry is consistent with that provided on the ECN website [6]. Specifically, the origin is located at the tip of the nozzle. The $z$-coordinate is positive in the direction of the spray, the $x$-coordinate is positive in the direction of the electrical connector, and the $y$-coordinate is transverse to the $x$-coordinate.

For nozzle tomography measurements, the beamline operated in "white beam" mode, which enables access to the unfiltered broadband (white) beam from the bending magnet source. This mode is necessary to image the internal nozzle geometry because the white beam provides increased flux at higher photon energies that are capable of penetrating through the dense steel. Figure 1 schematizes the experimental setup. Each Spray G nozzle 


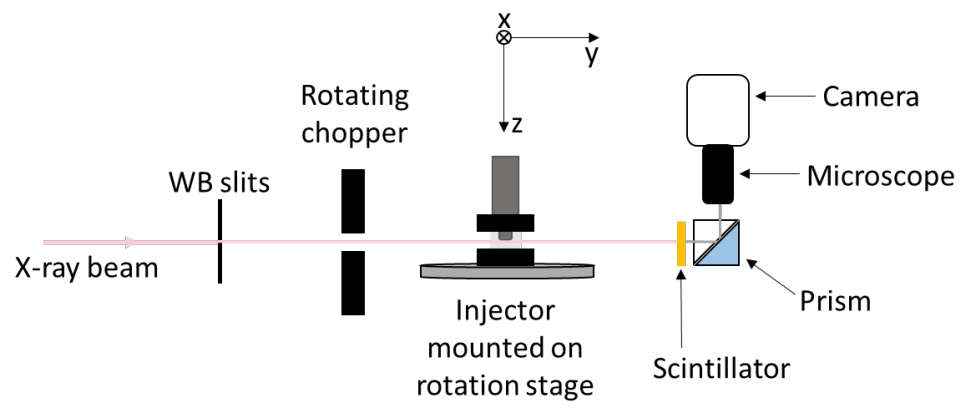

Figure 1. Schematic of the experimental setup for nozzle tomography (not to scale).

was mounted vertically in a specifically designed V-block holder that ensured nozzle stability during rotation. The holder was secured to two orthogonal stages that provided adjustment of the center of rotation. The pair of linear stages were mounted on an air-bearing rotation stage. From the APS storage ring, the polychromatic $x$-ray beam passes through a set of white beam slits that delimit the footprint of the x-ray source. Downstream of the slits, two metal filters, a $250 \mu \mathrm{m}$ copper and subsequent $25 \mu \mathrm{m}$ molybdenum filter, are placed in the beam path to reduce heat load on the nozzle. A rotating chopper downstream of the filters further minimizes the heat load by limiting beam exposure to $10 \%$ duty cycle, synchronized with the camera's frame rate. As the beam passes through the nozzle tip, x-ray photons are absorbed by the metal, creating spatial contrast in the beam cross-section. The outgoing $x$-ray photons that penetrated through the nozzle are absorbed by a lutetium aluminum garnet (LuAg) scintillator, and re-emitted as visible light. The propagation distance between the nozzle and scintillator was reduced to $48 \pm$ $1 \mathrm{~mm}$ in order mitigate phase effects that would otherwise increase the uncertainty in the geometry measurements by blurring the air/nozzle interface. The output image from the scintillator is magnified with a $5 x$ long-distance microscope to a field of view (FOV) of $2.25 \times 1.4 \mathrm{~mm}$ and pixel resolution of $1.17 \mu \mathrm{m}$. The diffraction-limited resolution of the raw images, determined by imaging an Xradia resolution target in the same tomography setup, is $1.5 \mu \mathrm{m}$. For nozzle tomography measurements, $\theta=0^{\circ}$ corresponds to the electrical connector pointing in the $+x$ direction. Each nozzle was rotated from $0^{\circ}$ to $180^{\circ}$ at $0.1^{\circ}$ steps, with 5 images recorded and subsequently averaged at each angle to increase the image contrast. Additional information with regard to the optimization of the imaging process may be found in previous work [30]. In brief, the process has been iteratively improved in order to minimize image manipulation during post-processing, and consequently preserve as much as possible the spatial resolution of the raw images. The resulting image stack undergoes tomographic reconstruction to transform the nozzle projections into $x-y$ slices through the nozzle body. The software that was used to perform the reconstruction is TomoPy, an open-source Python code developed for synchrotron tomography [31]. The reconstruction algorithm is a direct Fourier-based method called Gridrec [32]. The spatial uncertainty associated with the full imaging and post-processing procedure is $1.8 \mu \mathrm{m}$, determined from calibration of three steel pin gauges ranging in diameters between approximately 100 and $250 \mu \mathrm{m}$.

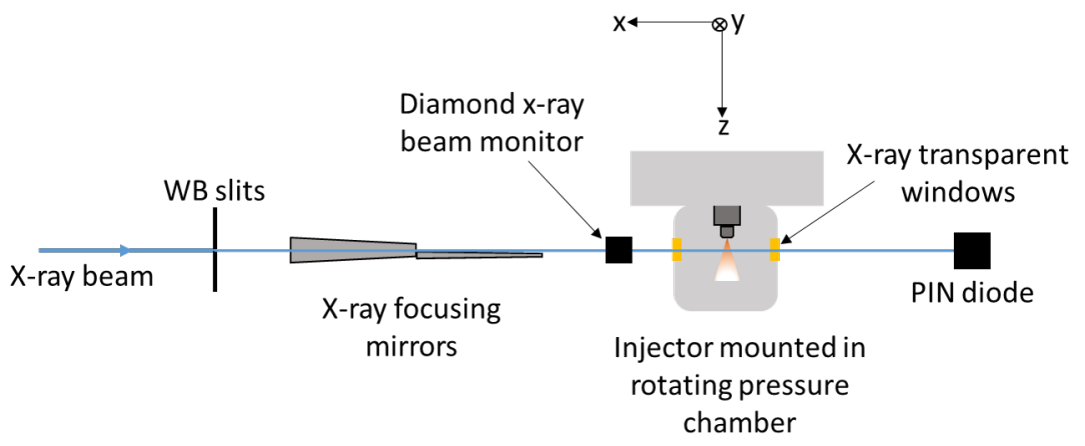

Figure 2. Schematic of the experimental setup for spray tomography (not to scale). The pressure chamber is shown as being transparent to visualize the location of the spray and $x$-ray transparent windows.

Spray tomography measurements were carried out using a monochromatic beam at $8 \mathrm{keV}$ energy. The working fuel was a gasoline surrogate, Viscor 1487, doped with 3.1 wt\% Rhodia DPX9 cerium additive to increase the signalto-noise ratio. The density and viscosity of the fuel, measured by a third party laboratory, are $810.1 \mu \mathrm{gm}^{-3}$ and $1.240 \mathrm{~mm}^{2} \mathrm{~s}^{-1}$ at standard temperature and pressure.

A schematic of the experimental setup is shown in Figure 2. Each injector was mounted vertically, spraying down, in a steel pressure chamber that allows for the injector's full rotation. The injection pressure was maintained at approximately $19 \mathrm{MPa}$ by a piston accumulator system. The back pressure was held constant at $0.3 \mathrm{MPa}$, and the 
electronic injection duration was set to $680 \mu \mathrm{s}$. The injector fired at $3 \mathrm{~Hz}$ into an ambient temperature environment that was pressurized with $\mathrm{N}_{2}$, which also provided a constant purge flow of 4 standard $L$ min $^{-1}$ in order to inhibit fuel droplet formation on the windows during data acquisition. The x-ray source was focused to a beam size of 5 $\times 6 \mu \mathrm{m}$. A transverse scan was carried out $2 \mathrm{~mm}$ downstream from the nozzle tip for $0^{\circ} \leq \theta \leq 180^{\circ}$ at $2^{\circ}$ steps. A total of 113 points spaced $80 \mu \mathrm{m}$ apart were measured at each rotation angle with a temporal resolution of 3.68 $\mu \mathrm{s}$. The outgoing beam intensity was averaged for 16 spray events at each measured position. The upstream beam intensity, $I_{0}$, was measured using a diamond x-ray beam monitor. As the beam passes through the spray, it becomes attenuated to an intensity $I$ by the fuel in the beam path on account of photoelectric absorption. The attenuation of the beam is related to the amount of fuel in the beam path via the Lambert-Beer law, which provides a measure of the projected density, $M$, of fuel at the measured location [15],

$$
\mathrm{M}\left(\mu \mathrm{g} \mathrm{mm}^{-2}\right)=\frac{1}{\mu} \log \left(\frac{I_{0}}{I}\right),
$$

where $\mu\left(\mathrm{mm}^{2} \mu \mathrm{g}^{-1}\right)$ is the mass absorption coefficient, found through calibration of the working fluid. The projected density measurements at the recorded 90 lines of sight are reconstructed using TomoPy's penalized maximum likelihood algorithm with weighted linear and quadratic penalties [33]. The result of the reconstruction is a time-resolved ensemble-average density field, $\rho(x, y, t)$, at $z=2 \mathrm{~mm}$ downstream of the nozzle tip. It should be noted that in this experiment setup, $\theta=0^{\circ}$ corresponded to the electrical connector pointing upstream to the beam propagation direction, as opposed to the nozzle tomography setup, in which the electrical connector was orthogonal to the beam direction. The inconsistency in the $\theta=0^{\circ}$ position relative to the beam between the spray and nozzle tomography experiments was accounted for in the analysis to ensure that the nozzle holes were properly indexed between the two measurement techniques.

\section{Data Analysis}

To remove background noise from the density field measurements, values that were less than $10 \%$ of the maximum density were masked at all time steps and all measured locations. In general, this threshold omitted approximately $2.5 \%$ of the total mass in the field from the analysis. In order to measure the fuel density corresponding to each hole, local minima in the density field were determined between holes by azimuthally resolving the steady-state density field. Wedge-shaped bins extending from the spray's steady-state center of mass through the local density minimum were used to isolate the density field associated with each nozzle hole (see Figure 4). The binning procedure is collectively exhaustive in $\theta$. The density field in each bin was integrated in both the $x$ - and $y$ - coordinate to arrive at the planar integrated mass (PIM),

$$
\operatorname{PIM}\left(\mu \mathrm{g} \mathrm{mm}^{-1}\right)=\int_{A} \rho(x, y, t) \mathrm{d} A \text {. }
$$

Ten dimensions of interest were measured per each nozzle hole. Figure 3 displays the relevant dimensions superposed on the isosurface of a Spray $G$ injector to illustrate the high spatial resolution of the measurements. The measured radii of curvature, lengths, diameters, and the drill angle are denoted by prefixes "R", "L", "D", and "A", respectively. The numbering scheme of the dimensions starts at 1 from the sac exit and successively increases in the direction of flow. The drill angle is calculated by tracking the centroid through the hole, and calculating the angle between the line through the center of mass (dashed in Figure 3) and the $z$-axis.

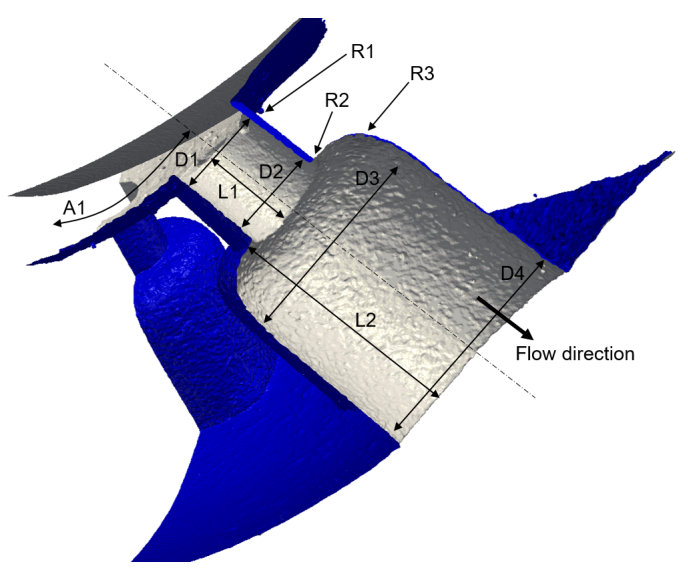

\begin{tabular}{l|l} 
ID & Feature $(\boldsymbol{\mu m})$ \\
\hline D1 & Hole inlet diameter \\
\hline D2 & Hole outlet diameter \\
\hline R1 & Hole inlet corner radius \\
\hline R2 & Hole outlet corner radius \\
\hline L1 & Hole length \\
\hline A1 & Drill angle \\
\hline D3 & Counterbore upstream diameter \\
\hline D4 & Counterbore downstream diameter \\
\hline R3 & Counterbore fillet \\
\hline L2 & Counterbore length
\end{tabular}

Figure 3. Measured hole dimensions of Spray G from x-ray nozzle tomography results.

In order to measure the dimensions summarized in Figure 3, each hole was individually rotated so that its horizontal axis aligned with the $x$-axis. A canny edge detector with a built-in Gaussian filter of specified standard deviation $\sigma=3$ was used to locate the hole cross-section at each slice along the $z$-plane [34]. A least-squares 
ellipse fitting algorithm $[35,36]$ was then used to measure the hole diameter and eccentricity. In general, because the eccentricity of the holes was typically less than the spatial resolution, an average of the diameters along the major and minor axes is quoted as the hole diameter. Any pixel location where the root-mean-square (RMS) error of the elliptic fitting exceeded 20 was removed from the analysis. The total uncertainty in the hole diameter measurements includes the spatial resolution, the RMS error associated with the elliptic fitting, and the error associated with approximating the cross-section as a perfect circle of 0 eccentricity. This error was typically dominated by the spatial resolution of $1.8 \mu \mathrm{m}$. In order to locate the hole inlet, a data point near the center of the hole was chosen as a starting point for a running average of the diameter, moving in the direction of the hole inlet. At each pixel location, the running average was updated, and the location of the hole inlet was defined as the point at which the diameter exceeded $3 \%$ of the running mean. A similar procedure was used to locate the hole and counterbore outlets. This technique proved successful in capturing the hole inlet and outlet locations because of the relatively cylindrical diameter profiles of both the hole and counterbore. The $3 \%$ threshold was chosen due to its ability to qualitatively capture the actual inlet and outlet locations. The hole outlet radius of curvature as well as the counterbore fillet were determined by fitting a 5th order polynomial to the hole corner profile and fillet, respectively. The radius of curvature was calculated using,

$$
C(\mu \mathrm{m})=\frac{\left(1+y^{\prime 2}\right)^{3 / 2}}{y^{\prime \prime}}
$$

where $y^{\prime}$ and $y^{\prime \prime}$ are the first and second derivatives of the fitted polynomial, respectively [29]. The radius of curvature corresponding to the corner was taken to be equal to $C_{m i n}$. Because it more accurately captured the curvature, a 6th order polynomial fit was used to model the hole inlet corner profile. Even though nominal dimensions are quoted in Table 1, it should be made clear that this work does not make an effort to compare measured to ideal dimensions, on account of the fact that the thresholds used to obtain the latter are unknown. For this reason, comparison between measured and nominal dimensions remains a subject for future work. The important point to note is that the same thresholds and methods of calculating nozzle dimensions are applied to all eight nozzles in order to maintain any errors associated with the data analysis as systematic errors, which would in turn not affect the relative trends in geometric variability.

Multiple linear regression analysis was used to fit a linear relationship between the steady-state PIM and the measured nozzle dimensions. Each nozzle hole was treated as a sample point, allowing statistics to be carried out on $n=64$ observations. The PIM was averaged during the steady portion of the spray, between $0.5<t<0.7 \mathrm{~ms}$. The general model for multiple linear regression, given $n$ observations, is,

$$
y_{i}=\beta_{0}+\beta_{1} x_{i, 1}+\beta_{2} x_{i, 2}+\ldots \beta_{m} x_{i}+\epsilon_{i} \text { for } i=1,2, \ldots n \text {, }
$$

where $y_{i}$ is the predictor variable (the average PIM), $x_{1} \ldots x_{m}$ are the explanatory variables (the dimensions listed in Figure 3), $\beta_{0} \ldots \beta_{i}$ are the coefficients, and $\epsilon$ is a normally distributed random variable to account for any noise in the data. To solve for the coefficients, a least-squares approach is used wherein the linear regression model attempts to minimize the sum of squared residuals between the observed and predicted values. In essence, this model attempts to describe the average PIM as a linear weighted function of the nozzle dimensions. The value of the adjusted coefficient of determination, $R^{2}$, is used to assess goodness of fit as well as to indicate whether or not the addition of a geometric feature improves the overall predictability of the model.

\section{Results and discussion}

To illustrate typical analysis results of spray tomography measurements as well as the binning procedure, Figure 4 plots the mean steady-state density field, $\bar{\rho}(x, y, t)$, for Spray $\mathrm{G} \# 12$, with and without masking density values that fall below a specified $10 \%$ threshold. All measured Spray G nozzles feature a well-atomized fuel spray at $2 \mathrm{~mm}$ downstream from the nozzle tip, with typical maximum liquid volume fractions of approximately 0.03 , as observed in previous work [7].

Figure 5a plots the PIM for all eight Spray G nozzles, in which the time of commanded start of injection occurs at $t=0 \mathrm{~ms}$. There is some variability in the apparent start and end of injection, as evidenced by the temporal spread of the PIM profiles. The apparent start of injection and the injection slope during the needle opening stage are not a strong function of injection pressure in GDI nozzles [37]. Aside from geometric variability, this may potentially be attributed to variable needle lift behavior. Needle motion measurements using $x$-ray phase contrast imaging at the APS have been previously obtained for Spray G \#28 [8]. It would be instructive to perform such measurements on the current injector set; this endeavor remains a topic for future work. In addition to the temporal spread, the PIM of Spray G \#22 is noticeably lower than that of the other injectors. Nevertheless, the variability in the steady-state PIM across all injectors is $2.4 \%$, which seems minor. Both Spray G \#12 and \#17 exhibited repeatable injector dribble after the end of injection. Figure $5 \mathrm{~b}$ displays this injector dribble, as well as hole-to-hole mass variations, for Spray G \#17. The hole numbering is consistent with that provided on the ECN website [6]. The mean variability in the hole-to-hole PIM for all eight injectors is $4.2 \%$. Table 1 provides a summary of the statistics for each measured dimension, calculated by treating each nozzle hole as an independent observation, i.e. with $n=64$ samples. In order to compare the variation from the mean value, we define the relative standard deviation (RSD) as,

$$
\mathrm{RSD}=\sigma / \mu,
$$




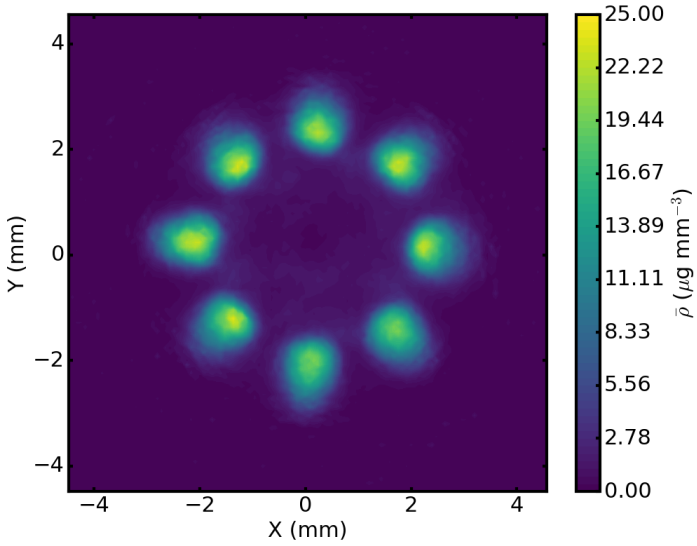

(a) Unmasked mean density field.

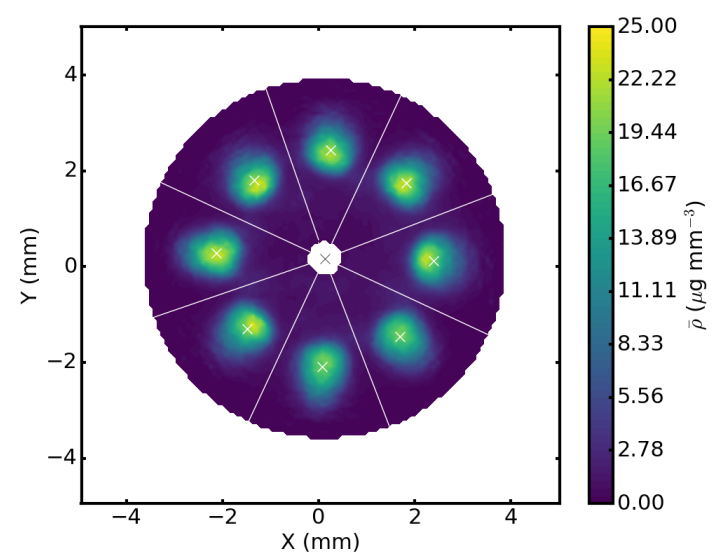

(b) Masked mean density field (mask is depicted by the white region). The crosses mark the center of mass of the holes (white) and the bulk spray (black). The data are binned into eight collectively exhaustive bins depicted by the white lines emanating radially from the center.

Figure 4. The mean density field for Spray G \#12, temporally averaged between 0.5 and $0.7 \mathrm{~ms}$.

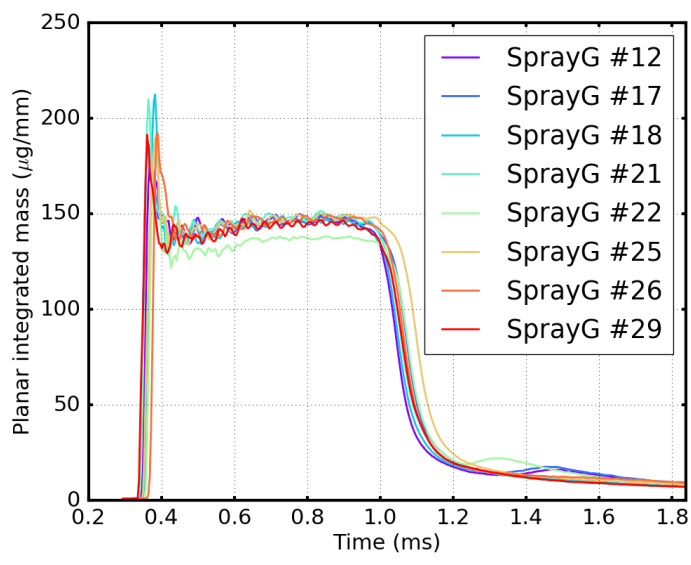

(a)

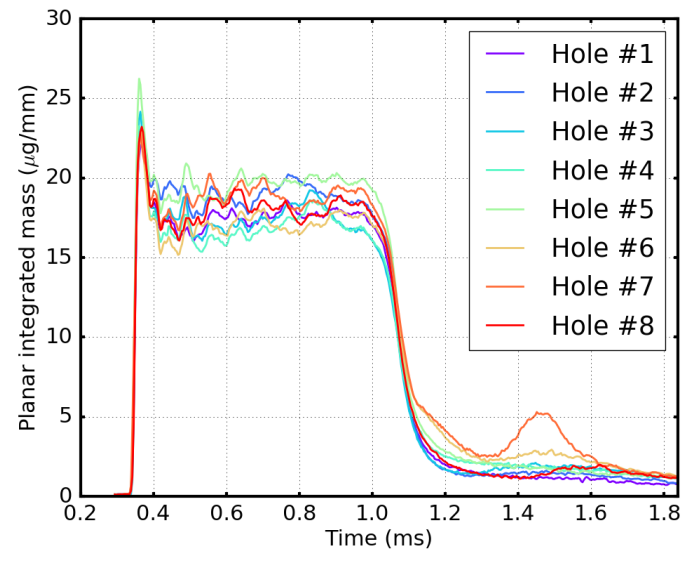

(b)

Figure 5. Planar integrated mass as a function of time for (a) all eight Spray G nozzles and (b) Spray G \#17.

where $\mu$ and $\sigma$ are the sample mean and standard deviation, respectively. This parameter shows the variability in relation to the mean, allowing for comparison among nozzle dimensions that vary in magnitude.

Generally, the largest variability is among the hole inlet and outlet corner radii. Table 2 lists the results of the multiple linear regression analysis, as well as the standard errors. $33.2 \%$ of the variability in the mean mass can be explained by variations in the inlet hole corner radius, the hole length, and the counterbore upstream diameter. The hole inlet corner radius has the strongest correlation with PIM variation. Because the coefficient is positive, the PIM is predicted to increase with increasing inlet hole radius. Simulations using the nominal geometry showed that the inlet corners can produce a pressure drop high enough for vapor formation [8]. However, the nominal geometry mesh features very sharp corners, and it is unknown whether this effect would still occur using a modified mesh with the prescribed dimensions listed in Table 1. In addition, inspection of select isosurfaces reveals voids at the hole inlet as large as $10 \mu \mathrm{m}$ in diameter, which can perturb the internal flow as the fuel enters the hole [38]. Increasing the inlet hole corner radius is expected to decrease the propensity for cavitation formation, which would in turn increase the discharge through the hole, in agreement with the regression analysis. The existence of vapor in the holes may also explain the temporal fluctuations in the PIM during steady injection, as shown in Figure 5 [8]. Time-resolved measurements of the needle motion and injection rate would provide further insight into this notion. The regression model also predicts that a larger counterbore upstream diameter decreases the mean steady-state mass. A potential reason for this may be increased area for mixing of fuel with ingested ambient gas inside the counterbore. Lastly, hole length is positively correlated with the mean PIM. An increase in hole length results in a larger ratio of length to diameter (L/D), which has been previously shown to suppress atomization [39]. 
Table 1. Hole dimensions measured by $\mathrm{x}$-ray nozzle tomography, as well as nominal dimensions from the manufacturer.

\begin{tabular}{c|cccc}
\hline Feature ID & Mean $(\mu \mathrm{m})$ & Standard deviation $(\mu \mathrm{m})$ & RSD $(\%)$ & Nominal $(\mu \mathrm{m})$ \\
\hline D1 & 175 & 1.14 & 0.65 & 165 \\
D2 & 176 & 1.00 & 0.57 & 165 \\
R1 & 4.93 & 0.88 & 18 & 0 \\
R2 & 2.54 & 0.47 & 18 & 0 \\
L1 & 150 & 10.50 & 7.0 & 170 \\
A1 & 38 & 0.47 & 1.3 & 37 \\
D3 & 394 & 1.29 & 0.33 & 388 \\
D4 & 394 & 1.27 & 0.32 & 388 \\
R3 & 65 & 3.30 & 5.1 & 40 \\
L2 & 402 & 11.18 & 2.8 & 470 \\
\hline
\end{tabular}

Table 2. Regression coefficients and standard errors.

\begin{tabular}{c|cc}
\hline Predictor Variable & Value & Standard error \\
\hline Constant, $\beta_{0}$ & 17.90 & 0.095 \\
R1, Hole inlet corner radius & 0.43 & 0.110 \\
L1, Hole length & 0.020 & 0.009 \\
D3, Counterbore upstream diameter & -0.21 & 0.074 \\
\hline
\end{tabular}

\section{Conclusions}

Measurements of the time-resolved density field at $z=2 \mathrm{~mm}$ downstream of the nozzle tip were obtained for eight nominally duplicate eight-hole Spray G nozzles using x-ray spray tomography. In addition, critical hole dimensions were retrieved from geometry measurements obtained by x-ray nozzle tomography. Both experiments were carried out at the 7-BM beamline of the APS at Argonne. Analysis of the PIM of each nozzle revealed subtle variations in the apparent start and end of injection timing among the injectors, as well as injector dribble from a few holes for two of the injectors. A multiple linear regression model was implemented to model the variability in the mean hole mass as a function of the hole's geometric features. Approximately $33 \%$ of the variation in the steady-state mass can be attributed to variations in the hole inlet radius of curvature, hole length, and counterbore upstream diameter. The remaining measured dimensions did not improve the predictability of the model. In general, the variability in the nozzle dimensions was low except for the hole inlet and outlet corner radii.

There are a variety of avenues to explore with regard to the future direction of this analysis. The strength of the statistical model may be improved by the addition of more Spray G nozzle holes, specifically targeting outlier injectors that may be expected to feature large variability. Integrating rate of injection and needle motion measurements into the analysis is also possible. The effect of nozzle upstream conditions on specific holes should be investigated by running an analysis that considers the spatial location of each hole, rather than treating all holes as independent observations. Considering the maximum and minimum dimensions in the analysis, as well as azimuthally resolving the inlet and outlet corner radii, may also prove useful in explaining more of the mass variability. In addition, quantifying surface roughness and the frequency of voids should be considered. Further data acquisition and data mining could explore such considerations in the future. At present, the analysis helps highlight which dimensions may need to be treated more carefully in both computational modeling efforts as well as in manufacturing practices, and conversely, which ones do not seem to strongly affect the ejected mass. Lastly, it should be noted that the linear regression model can only predict correlation, and not a cause-and-effect relationship. As such, the model findings will require further experimental and numerical investigation to prove causality between the relevant nozzle dimensions and fuel spray characteristics.

\section{Acknowledgments}

This research was performed at the 7-BM beamline of the APS at Argonne National Laboratory. Use of the APS is supported by the U.S. Department of Energy (DOE) under Contract No. DE-AC02-06CH11357. We gratefully acknowledge the computing resources provided on Blues, a high-performance computing cluster operated by the Laboratory Computing Resource Center at Argonne National Laboratory. We thank Dr. Doga Gürsoy for the use of TomoPy and corresponding user support, as well as Dr. Xianghui Xiao at the APS 2-BM beamline for technical guidance in performing x-ray tomography. Argonne's x-ray fuel injection research is sponsored by the DOE Vehicle Technologies Program under the direction of Gurpreet Singh and Leo Breton. 


\section{References}

[1] Duke, D.J., Swantek, A.B., Sovis, N., Tilocco, F.Z., Powell, C.F., Kastengren, A.L., Gürsoy, D. and Biçer, T., 2016, SAE Int. J. Engines, 9(1), pp. 143-152.

[2] Zhang, M., Drake, M.C., and Peterson, K., 2013, Proceedings of the ASME 2013 Internal Combustion Engine Division Fall Technical Conference.

[3] Sou, A., Minami, S., Prasetya, R.H., Moon, S., Wada, Y., and Yokohata, H., Aug 2015, 13th Triennial International Conference on Liquid Atomization and Spray Systems.

[4] Payri, R., Viera, J.P., Gopalakrishnan, V., and Szymkowicz, P.G., 2016, Fuel, 183, pp.20-33.

[5] Chaves, H. and Ludwig, Ch., 2005, Proceedings of the 20th ILASS Europe Meeting.

[6] Sandia National Laboratories. Engine Combustion Network "Spray G" injector plume orientation, https://ecn.sandia.gov/gasoline-spray-combustion/target-condition/spray-g-plume-orientation/ ([cit. 2017-03-07]).

[7] Strek, P., Duke, D., Swantek, A., Kastengren A., Powell, C.F., and Schmidt, D.P., 2016, SAE Technical Paper, 2016-01-0858.

[8] Baldwin, E.T., Grover Jr., R.O., Parrish, S.E., Duke, D.J., Matusik, K.E., Powell, C.F., Kastengren, A.L., and Schmidt, D.P., 2016, Int. J. Multiphase Flow, 87, pp. 90-101.

[9] Linne, M., 2013, Prog. in Energy and Comb. Sci., 39, pp. 403-440.

[10] Paryi, R., Salvador, F.J., Martí-Aldaravií, P., and Vaquerizo, D., 2017, Appl. Therm. Eng., 112, pp. 304-316.

[11] Aleiferis, P.G., Serras-Pereira, J., van Romunde, Z., Caine, J., and Wirth, M., 2010, Comb. and Flame, 157, pp.735-756.

[12] Mitroglou, N., Nouri, J.M., Gavaises, M., and Arcoumanis, C., 2005, Int. J. Engine Res. 7, pp. 255-270.

[13] Das, S., Chang, S.-I., and Kirwan, J., 2009, SAE Technical paper, 2009-01-1488.

[14] Payri, R., Gimeno, J., Marti-Aldaravi, P., and Vaquerizo, D., 2015, SAE Technical Paper, 2015-01-1893.

[15] Als-Nielsen, J. and McMorrow, D., 2011, "Elements of Modern X-Ray Physics". John Wily \& Sons.

[16] Lee, J.S., Weon, B.M., and Je, J.H., 2013, J. Phys. D: Appl. Phys., 46:494006.

[17] Kastengren, A.L., and Powell, C.F., 2014, Exp. Fluids, 55(3): 1686.

[18] Marchitto, L., Hampai, D., Dabagov, S.B., Allocca, L., Alfuso, S., Polese, C., and Liedl, A., 2015, Int. J. Multiphase Flow, 70, pp. 15-21.

[19] Bartolucci, L., Scarcelli, R., Wallner, T., Swantek, A., Powell, C.F., Kastengren, A., and Duke, D., 2016, SAE Technical Paper, 2016-01-0850.

[20] Swantek, A., Kastengren, A. Duke, D., Tilocco, Z. Sovis, N., and Powell, C.F., 2015, SAE Int. J. Fuels Lubr., 8(1), pp.160-166.

[21] Kastengren, A.L., Powell, C.F., Zunping, L., and Wang, J., 2009, SAE Technical Paper, 2009-01-0840.

[22] Cai, W., Powell, C.F., Yue, Y., Narayan, S., Wang, J., Tate, M.W., Renzi, M.J., Ercan, A., Fontes, E., Gruner, S.M., 2003, Appl. Phys. Lett. , 83(8), pp. 1671-1673.

[23] Liu, X., Cheong, S.-K., Powell, C.F., Wang, J., Hung, D.L.S., Winkelman, J.R., Tate, M.W., Ercan, A., Schuette, D.R., Koerner, L., Gruner, S.M., May 2005, 18th Annual Conference on Liquid Atomization and Spray Systems.

[24] Macian, V., Bermúdez, V., Payri, R., and Gimeno, J., 2003, Experimental Techniques, 27(2), pp.39-43.

[25] Kastengren, A.L., Tilocco, F.Z., Powell, C.F., Manin, J., Pickett, L.M., Payri, R. and Bazyn, T., 2012, Atomization and Sprays, 22(12), pp. 1011-1052.

[26] Feser, M., Gelb, J., Chang, H., Cui, H., Duewer, F., Lau, S.H., Tkachuk, A., and Yun, W., 2008, Meas. Sci. Technol., 19:094001.

[27] Wang, Y., De Carlo, F., Mancini, D.C., McNulty, I., Tieman, B., Bresnahan, J., Foster, I., Insley, J., Lane, P., von Laszewki, G., Kesselman, C., Su, MH., and Thiebaux, M., 2001, Rev. of Sci. Instr. 72(4), pp. 2062-2068.

[28] Pateyron, M., Peyrin, F., Laval-Jeantet, A.M., Spanne, P., Cloetens, P., and Peix, G., 1996, SPIE Med. Imag. 2708, pp.417-426.

[29] Kastengren, A., Powell, C.F., Arms, D., Dufresne, E.M., Gibson, H., and Wang, J., 2012, Journal of Synchrotron Radiation, 19(4), pp. 654-657.

[30] Matusik, K.E., Duke, D.J., Kastengren, A.L., Sovis, N., Swantek, A.B., and Powell, C.F., 2017, Int. J. Engine Res., submitted.

[31] Gürsoy, D., De Carlo, F., Xianghui, X., and Jacobsen, C., 2014, Journal of Synchrotron Radiation, 21(5), pp. 1188-1193.

[32] Marone, F. and Stampanoni, M., 2012, Journal of Synchrotron Radiation, 19(6), pp. 1029-1037.

[33] Chang, J.-H., Anderson, J.M.M., and Votaw, J.T., 2004, Medical Imaging, IEEE Transactions on, 23(9), pp.1165-1175.

[34] Canny, J., 1986, IEEE Trans. Pattern Analysis and Mach. Intel., 8, pp. 679-714.

[35] Fitzgibbon, A.W., Pilu, M., and Fischer, R.B., 1996, Proceedings of the 13th International Conference on Pattern Recognition, pp. 253-257.

[36] "Fitting an ellipse to a set of data points", http://nicky.vanforeest.com/misc/fitEllipse/fitEllipse.html ([cit. 2017-03-07]).

[37] Payri, R., Gimeno, J., Marti-Aldaravi, P., Vaquerizo, D., 2016, Atomization and Sprays, 26(9), pp. 889-919.

[38] Duke, D., Finney, C., Kastengren, A., Matusik, K., Sovis, N., Santodonato, L., Bilheux H., Schmidt, D., Powell, C., and Toops, T., 2017, SAE Technical Paper, 2017-01-0824.

[39] 2012, "Fuel Systems for IC Engines", edited by IMechE, Woodhead Publishing. 